\title{
AIN overgrowth of nano-pillar- patterned sapphire with different offcut angle by metalorganic vapor phase epitaxy
}

\author{
S. Walde ${ }^{*}, 1$, S. Hagedorn ${ }^{1}$, P.-M. Coulon ${ }^{2}$, A. Mogilatenko ${ }^{1}$, C. Netzel' ${ }^{1}$, J. Weinrich ${ }^{1}$, N. Susilo ${ }^{3}$, E. Ziffer ${ }^{3}$ \\ L. Matiwe ${ }^{4}$, C. Hartmann ${ }^{4}$, G. Kusch ${ }^{5}$, A. Alasmari', G. Naresh-Kumar ${ }^{5}$, C. Trager-Cowan ${ }^{5}$, T. Wernicke ${ }^{3}$, \\ T. Straubinger ${ }^{4}$, M. Bickermann ${ }^{4}$, R. W. Martin ${ }^{5}$, P. A. Shields ${ }^{2}$, M. Kneiss ${ }^{1,3}$, and M. Weyers ${ }^{1}$
}

${ }^{1}$ Ferdinand-Braun-Institut, Leibniz-Institut fuer Hoechstfrequenztechnik, Gustav-Kirchhoff-Str. 4, 12489 Berlin, Germany

${ }^{2}$ Centre of Nanoscience \& Nanotechnology, University of Bath, Bath, BA2 7AY, UK

Department of Electronic and Electrical Engineering, University of Bath, Bath, BA2 7AY, UK

Institute of Solid State Physics, Technical University Berlin, Hardenbergstr. 36, 10623 Berlin, Germany

${ }^{4}$ Leibniz-Institut fuer Kristallzuechtung, Max-Born-Str. 2, 12489 Berlin, Germany

${ }^{5}$ Department of Physics, SUPA, University of Strathclyde, 107 Rottenrow East, Glasgow G4 0NG, UK

Received ZZZ, revised ZZZ, accepted ZZZ

Published online ZZZ (Dates will be provided by the publisher.)

Keywords A3. Metal organic vapor phase epitaxy; B1. Nitrides; B1. Sapphire; B3. Light emitting diodes

\begin{abstract}
We present overgrowth of nano-patterned sapphire with different offcut angles by metalorganic vapor phase epitaxy. Hexagonal arrays of nano-pillars were prepared via Displacement Talbot Lithography and dry-etching. 6.6 $\mu \mathrm{m}$ crack-free and fully coalesced AlN was grown on such substrates. Extended defect analysis comparing X-ray diffraction, electron channeling contrast imaging and selective defect etching revealed a threading dislocation density of about $10^{9} \mathrm{~cm}^{-2}$. However, for c-plane sapphire offcut of $0.2^{\circ}$ towards $m$ direction the AlN surface shows step bunches with a height of $10 \mathrm{~nm}$. The detrimental
\end{abstract}

\begin{abstract}
impact of these step bunches on subsequently grown $\mathrm{AlGaN}$ multi-quantum-wells is investigated by cathodoluminescence and transmission electron microscopy. By reducing the sapphire offcut to $0.1^{\circ}$ the formation of step bunches is successfully suppressed. On top of such a sample an AlGaN-based UVC LED heterostructure is realized emitting at $265 \mathrm{~nm}$ and showing an emission power of $0.81 \mathrm{~mW}$ at $20 \mathrm{~mA}$ (corresponds to an external quantum efficiency of $0.86 \%$ ).
\end{abstract}

1 Introduction Due to the lack of bulk AlN substrates in sufficient numbers, diameters and low cost AlGaN-based ultraviolet (UV) light emitting diodes (LEDs) are typically grown on AlN/sapphire pseudo-substrates by metalorganic vapor phase epitaxy (MOVPE). This is motivated by numerous applications for UV LEDs, e.g. in phototherapy, UV curing and water disinfection ${ }^{1)-4)}$.

The main challenge in preparing AlN/sapphire templates is the high threading dislocation density (TDD) of about $10^{10} \mathrm{~cm}^{-2}$ at growth start due to the nucleation process and the difference in thermal expansion coefficients. During subsequent growth, these threading dislocations (TDs) penetrate through the layer stack in the growth direction leading to non-radiative recombination in the active region of subsequently grown LED heterostructures limiting the internal quantum efficiency $(\mathrm{IQE})^{5)}$. Experimental investigations and simulations by several groups have shown, that a reduction of the TDD below $10^{9} \mathrm{~cm}^{-2}$ will enable UV LEDs with high $\mathrm{IQE}^{6)-8)}$. To reach for such low TDD already in the AlN/sapphire template, there has been work on many different techniques in preparing AlN/sapphire templates as for example pulsed growth ${ }^{9}$, nitridation of the sapphire ${ }^{10)}$, high temperature annealing of AlN/sapphire ${ }^{11)-15)}$ and epitaxial lateral overgrowth (ELO) ${ }^{16)-18)}$ of AlN or sapphire patterns in the micrometer range. 
Further work on the ELO technique has shown that reducing the pattern dimensions to the nanometer range results in further advantages: Coalescence thickness shrinks cutting production costs ${ }^{19), 20)}$ and the TDD can be more efficiently reduced by bending many of the dislocation lines to the sidewalls of the structure ${ }^{21)}$. Besides material quality, using ELO especially with nano-patterns can enhance light extraction efficiency (LEE) for UV LEDs by suppressing internal total reflection at the AlN/sapphire interface through which the light is usually coupled out ${ }^{22), 23)}$.

There has been much work on nano-hole patterns, however only little on the inverted pattern of nano-pillars. Nano-patterned sapphire substrates (NPSS) with nano-pillars have the advantage that strain management is less critical, since AlN nucleates non-continuously on each pillar and later coalesces. Air-voids will be included which help to relax tensile strain. However, up to now it was not possible to achieve an atomically smooth surface since the high growth temperature usually applied for realizing coalescence leads to formation of high step bunches ${ }^{22), 24)}$. These surface steps lead to compositional inhomogeneity in subsequently grown $\mathrm{AlGaN}^{25}$ ) and can be detrimental in terms of efficiency of the final UV LED devices.

To address this problem, in this study we investigate AlN growth on nano-pillar NPSS with different offcut angles of the sapphire substrate. The cost-efficient, reproducible fabrication of sub-micrometer surface structures homogeneously on whole wafers is still challenging. Hence, we introduce a relatively new and easy approach including Displacement Talbot Lithography (DTL) ${ }^{26), 27)}$. To qualify the AlN/NPSS templates as device substrates, luminescence characteristics and structural properties of subsequently grown AlGaN multi quantum wells (MQWs) emitting at $265 \mathrm{~nm}$ are investigated. Furthermore, a full UVC LED heterostructure emitting at $265 \mathrm{~nm}$ is realized.

\section{Experimental setup}

2.1 Fabrication of NPSS Figure 1(a) presents the fabrication process to create arrays of sapphire nano-pillars. A stack of two layers was spin-coated on 2-inch sapphire substrates; first a bottom antireflective coating (BARC) layer (WiDE® 30W - Brewer Science) and second, a highcontrast positive resist layer (Dow® Ultra-i 123 diluted with Dow ${ }^{\circledR}$ EC11 solvent). Displacement Talbot Lithography (PhableR 100, Eulitha) was then used to expose the resist through an amplitude mask having a hexagonal configuration of $550 \mathrm{~nm}$ diameter circular openings with a pitch of 1 $\mu \mathrm{m}$. Nano-holes were formed in the resist after exposure and development along with an undercut in the underlying BARC. Metal was then deposited via e-beam evaporation and lift-off achieved by soaking the wafer in the developer. The resulting array of nano-dots was transferred into the sapphire via an inductively coupled plasma (ICP) dry etch

Copyright line will be provided by the publisher system (Oxford Instruments System 100 Cobra). The experiments were performed with $\mathrm{Cl}_{2} / \mathrm{BCl}_{3} / \mathrm{Ar}$ flows of $5 / 50 / 5$ sccm, a temperature set to $5{ }^{\circ} \mathrm{C}$, a pressure of 8 mTorr, 100 $\mathrm{W}$ RF power and $600 \mathrm{~W}$ ICP source power. Finally, the metal mask was removed in aqua-regia solution $\left(\mathrm{HCl}: \mathrm{HNO}_{3}\right.$, 3:1). Figure 1(b) and 1(c) display a tilted and a cross-sectional scanning electron microscopy (SEM) image of the nano-pillar NPSS after the overall process. The nano-pillars were found to be uniform across the 2 -inch wafer, with a flat top $c$-plane, a height of $260 \mathrm{~nm}$, and a top and bottom diameter of 280 and $430 \mathrm{~nm}$, respectively (sidewall facets tilted by $75^{\circ}$ towards the sapphire $c$-plane). This process was carried out for sapphire substrates with both $0.1^{\circ}$ and $0.2^{\circ}$ offcut towards the $m$ direction.
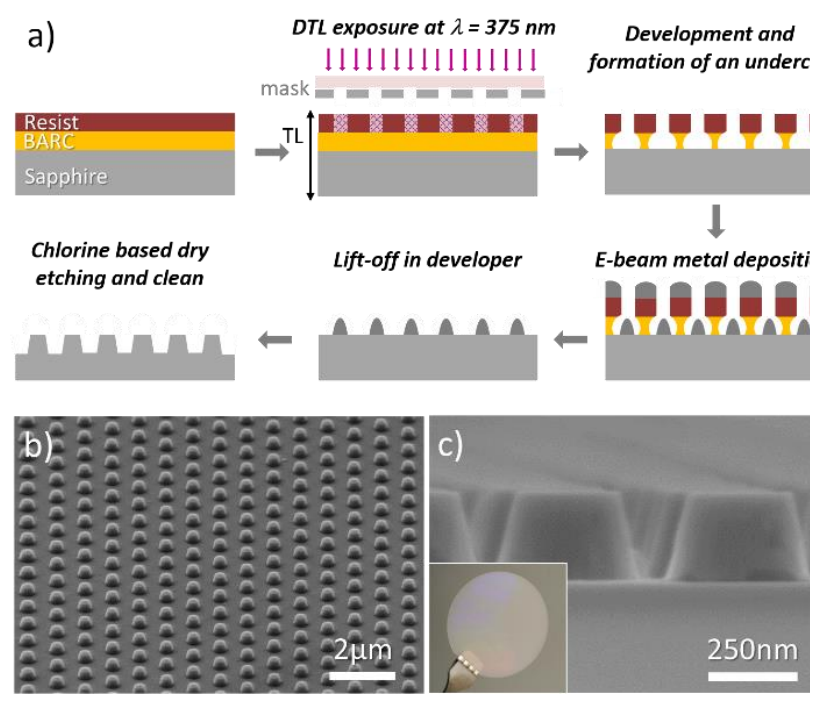

Figure 1 a) Sketch of the process flow for fabrication of nanopillar NPSS. b) Tilted view and c) cross-sectional SEM image of fully fabricated nano-pillar NPSS with photograph of full 2inch wafer as insert of c). ${ }^{28)}$

2.2 MOVPE growth and characterization For AlN growth on the NPSS with different offcut, an AIX2400G3HT MOVPE planetary reactor with a capability of 11 x 2-inch wafers was used with the standard precursors TMAl and $\mathrm{NH}_{3}$. Reactor pressure during growth was kept constant at 50 mbar and $\mathrm{H}_{2}$ served as carrier gas. At growth start, a $50 \mathrm{~nm}$ thick nucleation layer was deposited at a process temperature $\left(\mathrm{T}_{\text {proc }}\right)$ of $980{ }^{\circ} \mathrm{C}$ and an input group $\mathrm{V}$ to group III (V/III) ratio of $4000^{29)}$. After nucleation, a high temperature growth step was performed at $\mathrm{T}_{\text {proc }}=1380{ }^{\circ} \mathrm{C}$, $\mathrm{V} / \mathrm{III}$ ratio of 30 and resulting growth rate of $1.7 \mu \mathrm{m} / \mathrm{h}$. The high temperature growth step was followed by a medium temperature growth step at $\mathrm{T}_{\text {proc }}=1180{ }^{\circ} \mathrm{C}, \mathrm{V} / \mathrm{III}$ ratio of 30 and resulting growth rate of $1.5 \mu \mathrm{m} / \mathrm{h}$. During both growth steps the partial pressures were kept constant at $\mathrm{P}_{\mathrm{NH} 3}=$ 0.2235 mbar, $\mathrm{P}_{\mathrm{TMAl}}=0.0069$ mbar and $\mathrm{P}_{\mathrm{H} 2}=49.7763$ mbar. For in-situ characterization, the $405 \mathrm{~nm}$ reflectance from the sample surface during AlN overgrowth was monitored by a LayTec EpiCurveTT in-situ metrology system while $\mathrm{T}_{\text {proc }}$ 
was measured pyrometrically at the backside of the substrate holder. Subsequently, AlGaN-based multi-quantumwells (MQWs) and UVC-LEDs with emission near $265 \mathrm{~nm}$ at room temperature were grown by MOVPE in a Close Coupled Showerhead system using standard precursors and dopants (TMGa, TEGa, TMAl, $\left.\mathrm{NH}_{3}, \mathrm{Cp}_{2} \mathrm{Mg}, \mathrm{SiH}_{4}\right)^{13), 30)}$. Nitrogen and hydrogen were used as carrier gases. On top of the AlN/NPSS templates a $400 \mathrm{~nm}$ thick homoepitaxial AlN buffer layer followed by a $900 \mathrm{~nm}$ thick $\mathrm{Al}_{0.76} \mathrm{Ga}_{0.24} \mathrm{~N}: \mathrm{Si}$ current spreader and a $200 \mathrm{~nm}$ thick $\mathrm{Al}_{0.65} \mathrm{Ga}_{0.35} \mathrm{~N}$ : Si contact layer were grown with the $\mathrm{Al}$ content between those two layers graded over $100 \mathrm{~nm}$. The active region consists of an $\mathrm{Al}_{0.48} \mathrm{Ga}_{0.52} \mathrm{~N} / \mathrm{Al}_{0.63} \mathrm{Ga}_{0.37} \mathrm{~N} \mathrm{MQW}$ stack. The electron blocking heterostructure consists of a 10 $\mathrm{nm}$ thick $\mathrm{Al}_{0.85} \mathrm{Ga}_{0.15} \mathrm{~N}$ interlayer and a $25 \mathrm{~nm}$ thick $\mathrm{Al}_{0.75} \mathrm{Ga}_{0.25} \mathrm{~N}: \mathrm{Mg}$ electron blocking layer. The heterostructure is capped with a $200 \mathrm{~nm}$ thick GaN:Mg contact layer. The MQW heterostructure is identical to the UVC-LED heterostructure except for the omission of the electron blocking heterostructure and the GaN:Mg contact layer Atomic force microscopy (AFM) was utilized to investigate the surface morphology of the AlN after overgrowth. X-ray diffraction (XRD) was used for measuring rocking curves with a Philips X'Pert Pro system including a four-fold $220 \mathrm{Ge}$ monochromator to estimate the TDD. The aperture on the source side was $0.5 \mathrm{~mm} \times 5 \mathrm{~mm}$ and the acceptance angle in front of the detector was $1^{\circ}$. Two additional ways to determine the TDD were applied: Selective defect etching by an alkaline melt ${ }^{31)}$ and electron channeling contrast imaging $(\mathrm{ECCI})^{32)-35)}$. To characterize the luminescence behavior, cathodoluminescence (CL) measurements of the AlGaN MQW s on top of the AlN were performed in plan-view. Furthermore, morphological inhomogeneities were investigated by cross-sectional scanning transmission electron microscopy (STEM). Finally, on-wafer electroluminescence (EL) measurements were performed for the full UVC-LED heterostructure using indium dots as contacts.

\section{Results and discussion}

3.1 AIN overgrowth To successfully grow on the NPSS, a three-step growth process was performed ${ }^{24)}$. In-situ $405 \mathrm{~nm}$ reflectance and temperature transients during growth on the $0.2^{\circ}$ and $0.1^{\circ}$ offcut samples are shown in Fig. 1. After low temperature nucleation, high temperature $\left(1380{ }^{\circ} \mathrm{C}\right)$ and low V/III ratio (30) were applied to enhance the diffusion length of $\mathrm{Al}$ adatoms and hence to favor lateral growth. The reflectance shows typical Fabry-Perot oscillations due to increasing layer thickness while the overall average reflectance increases and then saturates at a value of about 0.12 at a layer thickness of about $4 \mu \mathrm{m}$, indicating coalescence of the layer. The subsequent growth at medium temperature $\left(1180{ }^{\circ} \mathrm{C}\right)$ at the same V/III ratio (30) should shift the growth mode from step bunching growth towards step-flow growth mode to smoothen the surface after coalescence ending up with a total layer thickness of about 6.6 $\mu \mathrm{m}$. Both reflectance curves for the samples with different offcuts in principal follow the same behavior. However, a slightly higher average reflectance during medium temperature growth for the $0.1^{\circ}$ offcut sample can be observed.

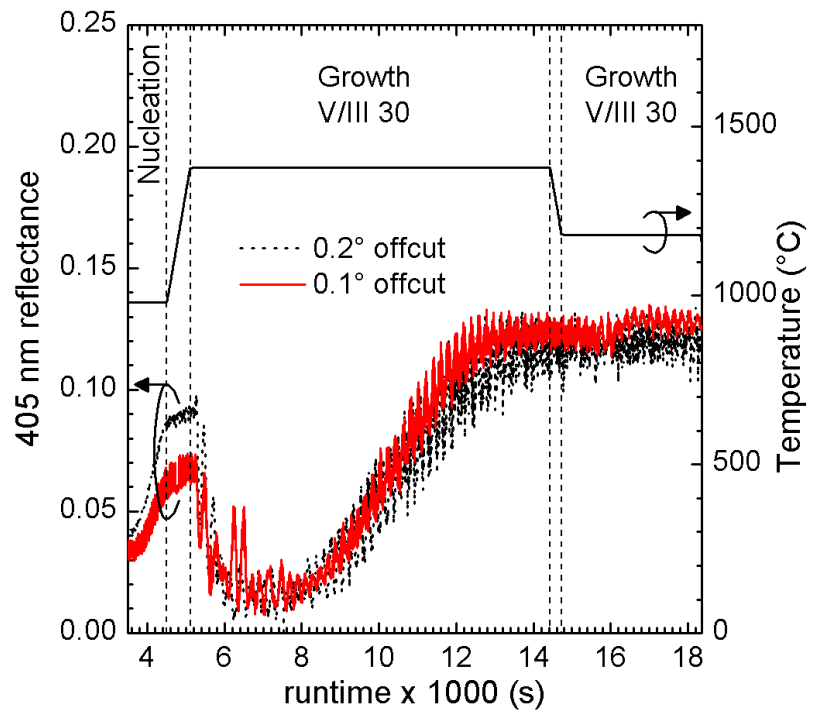

Figure $2405 \mathrm{~nm}$ reflectance and process temperature $\mathrm{T}_{\text {proc }}$ against run time for the three-step growth process on NPSS with offcut of $0.2^{\circ}$ (dashed-black) and $0.1^{\circ}$ (solid-red).

AFM images of the surface morphology for both samples before and after the medium temperature growth step to smoothen the surface are shown in Fig. 3. Before smoothing, there are step bunches visible for both samples (Fig. 3a,b) resulting in a rather rough surface with a root mean square (rms) roughness value of $3.7 \mathrm{~nm}$ for $0.2^{\circ}$ and $2.9 \mathrm{~nm}$ for $0.1^{\circ}$ offcut on $10 \mu \mathrm{m} \times 10 \mu \mathrm{m}$ surface area. After smoothing, the $0.2^{\circ}$ offcut sample still shows step bunches $(\mathrm{rms}=3.7 \mathrm{~nm})$ while for the $0.1^{\circ}$ offcut sample an atomically smooth surface $(\mathrm{rms}=0.9 \mathrm{~nm})$ with typical bilayer steps is observed (Fig. 3c,d). Step bunches form if the $\mathrm{Al}$ adatom diffusion length exceeds a certain value in relation to the terrace width determined by the sample offcut ${ }^{36), 37)}$. They formed for both samples during the high temperature growth step in which the $\mathrm{Al}$ adatom diffusion length is at its maximum. However, the medium temperature growth step induced step-flow growth mode for the $0.1^{\circ}$ offcut sample due to the larger terrace length leading to smoothing of the step bunches in contrast to the $0.2^{\circ}$ offcut sample for which the stepbunched growth prevails. This also explains the slight increase of the average reflectance during medium temperature growth for the $0.1^{\circ}$ offcut sample, since the step bunches lead to a small amount of scattering reducing the reflectance until they are smoothened. 


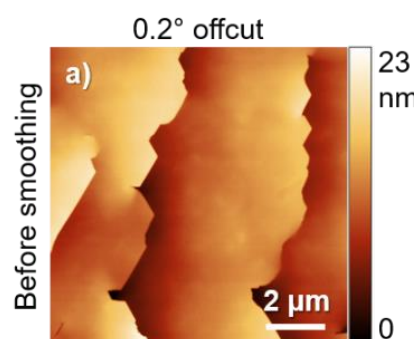

$\mathrm{rms}=3.6 \mathrm{~nm}$

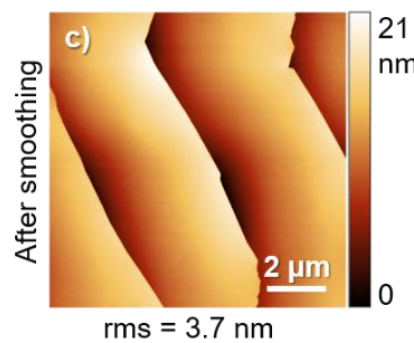

$\mathrm{rms}=3.7 \mathrm{~nm}$

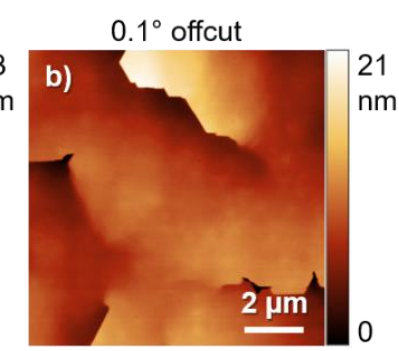

$\mathrm{rms}=2.9 \mathrm{~nm}$

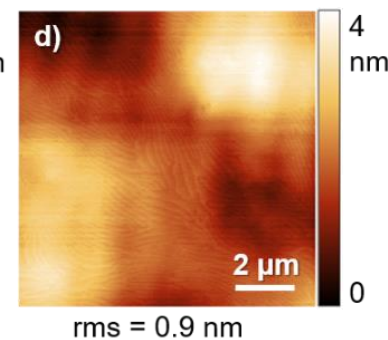

Figure 3 AFM images of the AlN surface before and after smoothing for NPSS with a), c) $0.2^{\circ}$ offcut and b), d) $0.1^{\circ}$ offcut NPSS including the associated rms value for comparison of the roughness.

3.2 Defect analysis For defect analysis, firstly, the standard technique of measuring the $\mathrm{X}$-ray rocking curve FWHM (XRC-FWHM) of the 0002 and 10-12 reflections was carried out. For the $0.2^{\circ}$ offcut sample values of 300 arcsec and 810 arcsec were acquired, for the $0.1^{\circ}$ offcut sample 510 arcsec and 1160 arcsec for 0002 and 10-12 respectively. That leads to estimated ${ }^{38), 39)}$ TDDs of $7 \times 10^{9} \mathrm{~cm}^{-2}$ for the $0.2^{\circ}$ and $1 \times 10^{10} \mathrm{~cm}^{-2}$ for the $0.1^{\circ}$ offcut sample. These are relatively high values for about $6.6 \mu \mathrm{m}$ AlN on sapphire. Unfortunately, the calculation of TDDs from XRC-FWHM can be applied only if there are no other significant broadening mechanisms present in AlN. In case of the laterally overgrown regions on the samples and due to the non-continuous surface at growth start, wing tilt is expected to broaden the XRC-FWHM ${ }^{24), 40)}$ and hence can falsify the TDD estimation by calculation. Therefore, alternative methods for analyzing the TDDs, namely ECCI and selective defect etching, were applied to both samples (Fig. 4). From ECCI (Fig. 4a,b), TDDs of $8 \times 10^{8} \mathrm{~cm}^{-2}$ for $0.2^{\circ}$ and $1.5 \times 10^{9} \mathrm{~cm}^{-2}$ for $0.1^{\circ}$ offcut were estimated by simply counting the dislocations (seen as points in black-white contrast $)^{32), 33)}$. For selective defect etching (Fig. 4c,d), TDDs of $9 \times 10^{8} \mathrm{~cm}^{-2}$ for $0.2^{\circ}$ offcut and $1 \times 10^{9} \mathrm{~cm}^{-2}$ for $0.1^{\circ}$ offcut were determined by counting the etch pits after etching. The smaller etch pits can be ascribed to pure edge type TDs while mixed type TDs yield bigger pits ${ }^{31), 41)}$. The small deviation in TDD for the $0.2^{\circ}$ offcut sample between ECCI and selective defect etching can be explained by leaving out dislocations at the step edges in the case of ECCI, since the topographic contrast from the step edges dominates the diffraction contrast required to image dislocations (Fig. 4a), while selective defect etching clearly shows many TDs lined up along the step edges (Fig. 4c). Attractive forces in the direction of free surfaces acting on TDs ${ }^{42)}$ lead to TD bending towards the step sidewalls during growth and therefore induce this lining up along the steps. For the uniformly distributed dislocations of the $0.1^{\circ}$ offcut sample, without overlapping contrast of surface steps, the TDDs determined by ECCI and selective defect etching are in good agreement. The slightly higher TDD of about $1 \times 10^{9} \mathrm{~cm}^{-2}$ for $0.1^{\circ}$ offcut in comparison to the $0.2^{\circ}$ offcut sample is related to the lining up of the TDs along the step edges, since this increases the probability of dislocations to annihilate. In all, it can be concluded, that the much lower TDDs suggested by ECCI and selective defect etching are more trustworthy than the TDDs calculated from the XRD FWHM.

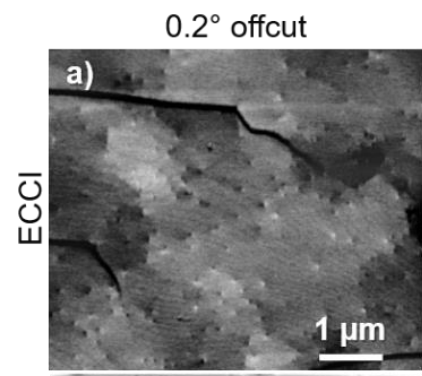

$0.1^{\circ}$ offcut

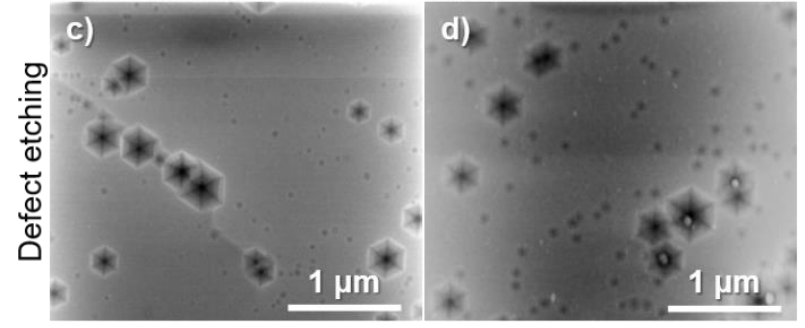

Figure 4 ECCI images ${ }^{43}$ ) of the surface (after smoothing) for the a) $0.2^{\circ}$ and b) $0.1^{\circ}$ offcut sample and SEM images of the surface (after smoothing) after selective defect etching for the c) $0.2^{\circ}$ and d) $0.1^{\circ}$ offcut sample.

3.3 AIGaN heterostructure growth The AlN/NPSS templates were overgrown with AlGaN MQWs emitting at $265 \mathrm{~nm}$ at room temperature. Their luminescence behavior was investigated by spatially-resolved low-temperature CL at $80 \mathrm{~K}$ (Fig. 5a,b). The main luminescence peak was found at $255 \mathrm{~nm}$ and is attributed to the luminescence of $265 \mathrm{~nm}$ at room temperature. In the monochromatic CL images at $255 \mathrm{~nm}$ dark pits are observed for the $0.2^{\circ}$ offcut sample while for the $0.1^{\circ}$ offcut sample the luminescence is homogenous. Some of the dark pits show luminescence at a longer wavelength of $318 \mathrm{~nm}$ (Fig. 5c). The pits themselves seem to be aligned in lines following the arrangements of the former step bunches on the $0.2^{\circ}$ offcut AlN template. Crosssectional STEM investigations (Fig. 6) show that these Vshaped pits originate deep inside the AlGaN. They are formed above TDs (Fig. 6b) and are partly overgrown during the subsequent growth. Probably, similar to InGaN alloys, they can form out of TDs with a large Burgers vector 
such that the building up of free surfaces is energetically favorable ${ }^{44)-46)}$.

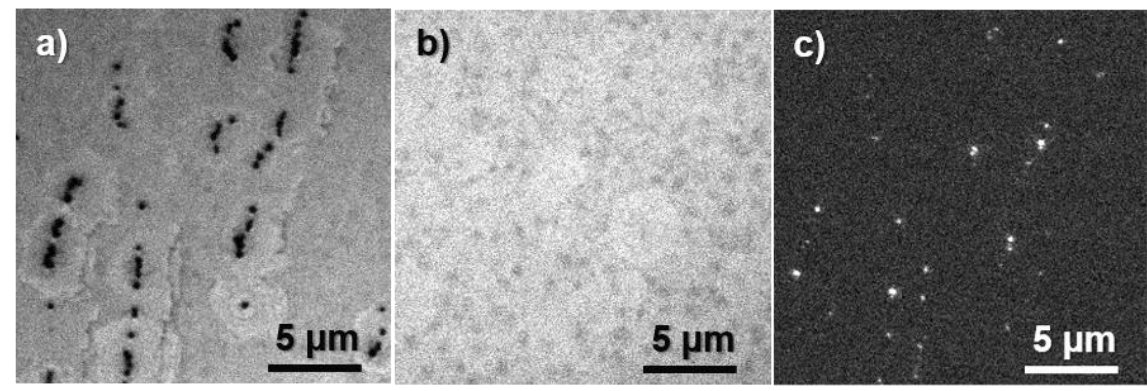

Figure 5 Mono-CL images (plan-view) acquired at $255 \mathrm{~nm}$ of the AlGaN MQWs for the a) $0.2^{\circ}$ and b) $0.1^{\circ}$ offcut sample. c) MonoCL image at $318 \mathrm{~nm}$ at the same position of the $0.2^{\circ}$ offcut sample as in a).
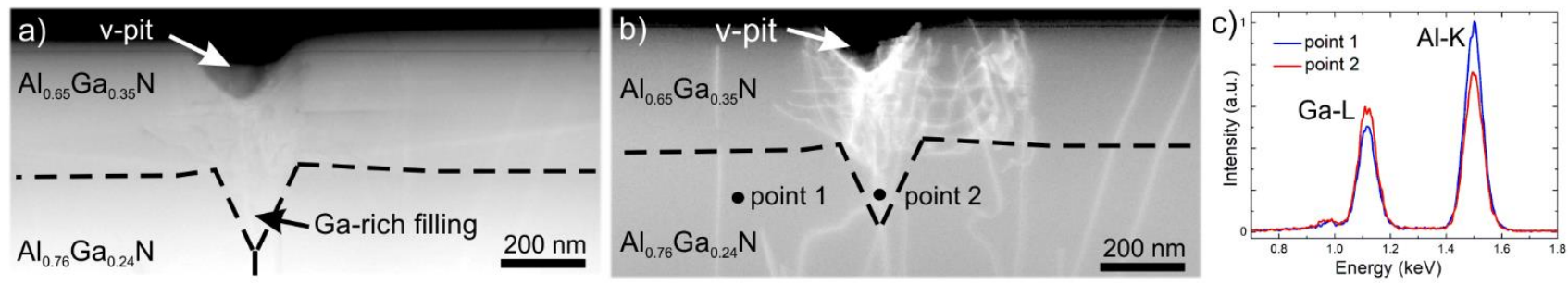

Figure 6 a) Cross-sectional STEM image of a V-pit in the layer stack on the $0.2^{\circ}$ off-cut sample. b) Annular dark-field STEM image of the same sample position showing that the V-pit formation occurs above a dislocation line. The subsequent V-pit filling with Ga-rich $(\mathrm{Al}, \mathrm{Ga}) \mathrm{N}$ leads to the formation of a broad dislocation net around the V-pit. c) EDX spectra acquired at the sample positions marked in (b) proving the higher Ga content in the V-pit.

This explains also the alignment along step edges, since as shown before, the TDs tend to line up there, increasing the probability of dislocation cluster formation with resulting large Burgers vectors. The luminescence at $318 \mathrm{~nm}$ (Fig. 5c) can be explained by preferential $\mathrm{Ga}$ incorporation on the sidewalls and inside these pits due to the higher diffusion length of the $\mathrm{Ga}$ adatoms during $\mathrm{AlGaN}$ growth ${ }^{25), 47), 48)}$ and the locally lower compressive strain in the vicinity of sidewall facets. Energy dispersive X-ray (EDX) spectra prove the increased Ga content inside such a V-pit compared to areas without any V-pits (Fig. 6c). Despite the presence of free surfaces inside the pits, which facilitate strain relaxation, the growth of Ga-rich material inside them leads to further strain relaxation by the formation of a broad net of dislocations around the pits (Fig. 6b). These defect-rich regions are spatially localized around the pits, but appear directly within the active region. Obviously, such pits will limit the final device performance of an LED by limiting mainly the IQE but also being possible short cuts leading to increased leakage current ${ }^{49}$.

Finally, a full UVC-LED heterostructure emitting at $265 \mathrm{~nm}$ was grown on top of the most promising $0.1^{\circ}$ offcut AIN/NPSS template. Electroluminescence measurements using on-wafer indium dot quick test show the spectrum with clear single peak emission at $265 \mathrm{~nm}$ (Fig. 7a). Furthermore, an emission power of $0.81 \mathrm{~mW}$ at $20 \mathrm{~mA}$ was meas- ured (Fig. 7b) corresponding to an external quantum efficiency of $0.86 \%$. This proves the possibility of using AIN/nano-pillar NPSS as UV-LED templates obtaining comparable results with respect to recently published LEDs from our groups using the different template technology of sputtered and high temperature annealed AlN/sapphire ${ }^{13)}$.

4 Conclusions In this work, nano-pillar NPSS with different offcut angles were successfully fabricated by Displacement Talbot Lithography and dry etching. Applying a three step growth process, they were overgrown ending up with $6.6 \mu \mathrm{m}$ crack-free and fully coalesced AlN. The sample with $0.2^{\circ}$ substrate offcut showed $10 \mathrm{~nm}$ high step bunches while for the lower offcut of $0.1^{\circ}$ the surface appears atomically smooth. An extended defect analysis showed that for these samples the estimation of TDD from XRC-FWHM fails, most probably due to wing tilt in the laterally grown AlN. ECCI and selective defect etching showed more reliable TDD determination for the samples: The AlN grown on nano-pillar NPSS shows TDDs of about $10^{9} \mathrm{~cm}^{-2}$. However, for the larger offcut of $0.2^{\circ}$, step bunches formed which accumulated an increased number of TDs and led to the formation of pits in subsequently grown $\mathrm{AlGaN}$ and thus inhomogeneous luminescence from the MQWs on top. In contrast, for the smaller offcut of $0.1^{\circ}$, the formation of step bunches was suppressed, and hence, homogeneous luminescence of subsequently grown AlGaN MQWs could be 
achieved. On such a sample, a full UVC-LED heterostructure emitting at $265 \mathrm{~nm}$ was realized showing an emission power of $0.81 \mathrm{~mW}$ at $20 \mathrm{~mA}$ corresponding to an external quantum efficiency of $0.86 \%$. This result is comparable to another state-of-the-art template technology of sputtered and high temperature annealed AlN/sapphire.
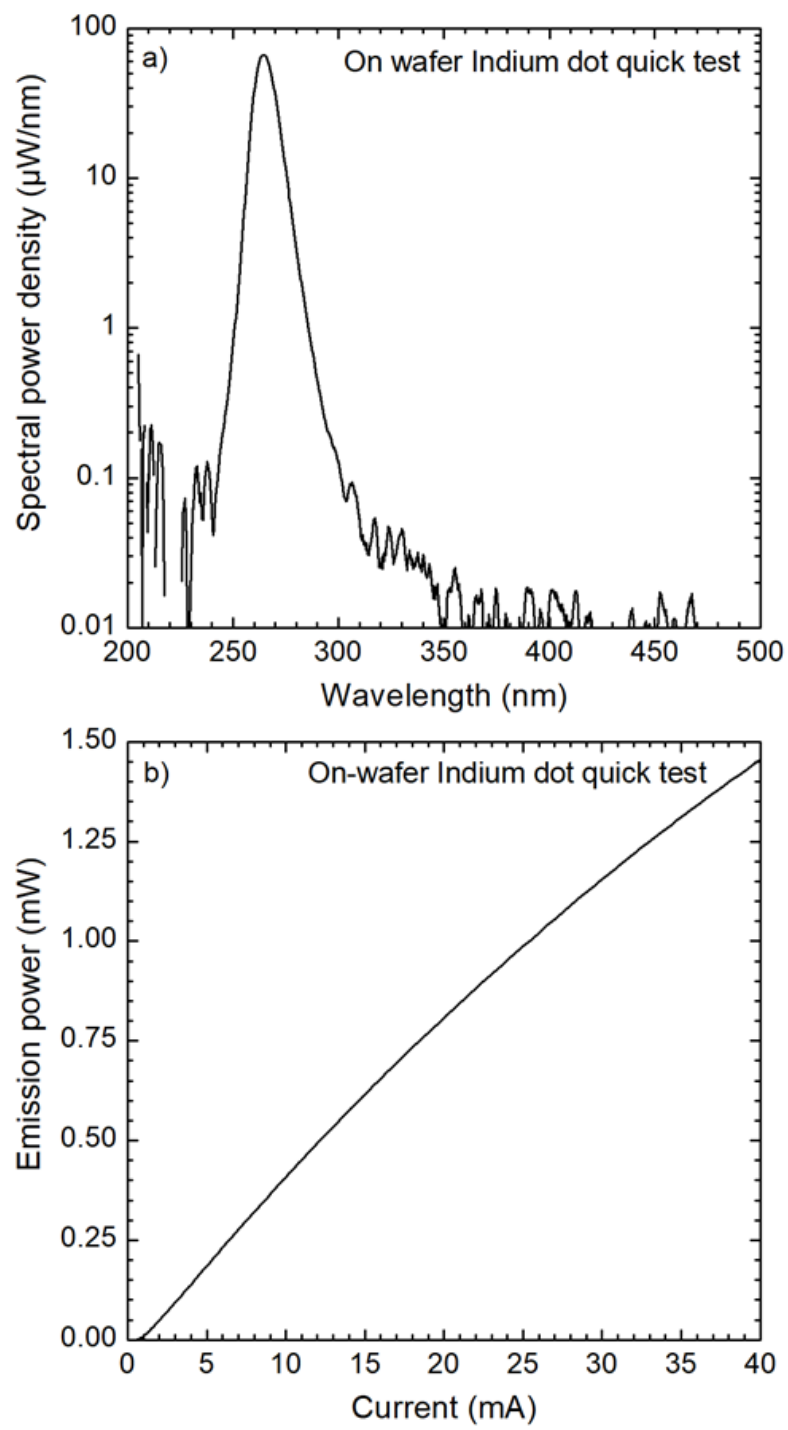

Figure 7 a) Representative emission spectrum at $20 \mathrm{~mA}$ and b) LI curve of the UVC-LED on the $0.1^{\circ}$ offcut AIN/NPSS sample.

Acknowledgements This work was partially funded by the German Federal Ministry of Education and Research (BMBF) within the Advanced UV for Life consortium and by the German Research Foundation (DFG) within the Collaborative Research Center "Semiconductor Nanophotonics" (CRC 787). It was also partially funded by the UK Engineering and Physical Sciences Research Council "Manufacturing of nano-engineered III-nitride semiconductors" programme, (EP/M015181/1, EP/M022862/1). Furthermore, the authors thank Torsten Petzke for technical assistance on the MOVPE machine. This publication is supported by multiple datasets, which are openly available at locations cited in the reference section.

\section{References}

[1] W. L. Morison and T. B. Fitzpatrick, Raven Press, New York, 1991.

[2] M. Kneissl and J. Rass, "III-Nitride ultraviolet emitters", in Springer Series in Materials Science (Springer, 2016).

[3] M. Würtele, T. Kolbe, M. Lipsz, A. Külberg, M. Weyers, M. Kneissl, and M. Jekel, Water Res. 45, 1481 (2011).

[4] H. Hirayama, T. Yatabe, N. Noguchi, and N. Kamata, Electron. Commun. Jpn. 93, 24 (2010).

[5] S. Y. Karpov and Y. N. Makarov, Appl. Phys. Lett. 81, 4721 (2002).

[6] M. Kneissl, T. Kolbe, C. Chua, V. Kueller, N. Lobo, J. Stellmach, A. Knauer, H. Rodriguez, S. Einfeldt, Z. Yang, N.M. Johnson, and M. Weyers, Semicond. Sci. Technol. 26, 014036 (2011).

[7] M. Kneissl, T.-Y. Seong, J. Han, and H. Amano, Nat. Photon. 13, 233 (2019).

[8] K. Ban, J.-I. Yamamoto, K. Takeda, K. Ide, M. Iwaya, T. Takeuchi, S. Kamiyama, I. Akasaki, H. Amano, Appl. Phys. Express 4, 052101 (2011).

[9] H. Hirayama, T. Yatabe, N. Noguchi, T. Ohashi, and N. Kamata, Appl. Phys. Lett. 91, 071901 (2007).

[10] M. Funato, M. Shibaoka, and Y. Kawakami, J. Appl. Phys. 121, 085304 (2017).

[11] H. Miyake, G. Nishio, S. Suzuki, K. Hiramatsu, H. Fukuyama, J. Kaur, and N. Kuwano, Appl. Phys. Express 9, 025501 (2016).

[12] H. Miyake, C.-H. Lin, K. Tokoro, and K. Hiramatsu, J. Crystal Growth 456, 155 (2016).

[13] N. Susilo, S. Hagedorn, D. Jaeger, H. Miyake, U. Zeimer, C. Reich, B. Neuschulz, L. Sulmoni, M. Guttmann, F. Mehnke, C. Kuhn, T. Wernicke, M. Weyers, and M. Kneissl, Appl. Phys. Lett. 112, 041110 (2018).

[14] S. Hagedorn, S. Walde, A. Mogilatenko, M. Weyers, L. Cancellara, M. Albrecht, and D. Jaeger, J. Crystal Growth 512, 142 (2019).

[15] S. Walde, S. Hagedorn, and M. Weyers, Jpn. J. Appl. Phys. 58, SC1002 (2019).

[16] H. Hirayama, S. Fujikawa, J. Norimatsu, T. Takano, K. Tsubaki, and N. Kamata, Phys. Stat. Sol. C 6, S356 (2009).

[17] V. Kueller, A. Knauer, U. Zeimer, H. Rodriguez, A. Mogilatenko, M. Kneissl, and M. Weyers, Phys. Stat. Sol. C 8, 2022 (2011).

[18] B. Tran, H. Hirayama, N. Maeda, M. Jo, S. Toyoda, and N. Kamata, Sci. Rep. 5, 14734 (2015).

[19] P. Dong, J. Yan, J. Wang, Y. Zhang, C. Geng, T. Wei, P. Cong, Y. Zhang, J. Zeng, Y. Tian, L. Sun, Q. Yan, J. Li, S. Fan, and Z. Qin, Appl. Phys. Lett. 102, 241113 (2013).

[20] L. Zhang, F. Xu, J. Wang, C. He, W. Guo, M. Wang, B. Sheng, L. Lu, Z. Qin, X. Wang, and B. Shen, Sci. Rep. 6,35943 (2016). 
[21] M. Conroy, V. Z. Zubialevich, H. Li, N. Petkov, J. D. Holmes, and P. J. Parbrook, J. Mat. Chem. C 3, 431 (2015).

[22] D. Lee, J. Lee, J. Jang, I.-S. Shin, L. Jin, J. H. Park, J. Kim, J. Lee, H.-S. Noh, Y.-I. Kim, Y. Park, G.-D. Lee, Y. Park, J. Kim, and E. Yoon, Appl. Phys. Lett. 110, 191103 (2017).

[23] Y. K. Ooi, and J. Zhang, IEEE Photonics J. 10, 8200913 (2018).

[24] S. Hagedorn, A. Knauer, A. Mogilatenko, E. Richter, and M. Weyers, Phys. Stat. Sol. A 213, 3178 (2016).

[25] A. Knauer, U. Zeimer, V. Kueller, and M. Weyers, Phys. Status Solidi C 11, 377 (2014).

[26] H. H. Solak, C. Dais, and F. Clube, Opt. Express 19, 10686 (2011).

[27] P.-M. Coulon, B. Damilano, B. Alloing, P. Chausse, S. Walde, J. Enslin, R. Armstrong, S. Vézian, S. Hagedorn, T. Wernicke, J. Massies, J. Zúñiga-Pérez, M. Weyers, M. Kneissl, and P. A. Shields, accepted for publication in Micro and Nanoengineering.

[28] P. Coulon, 2019. Dataset for "Overgrowth of nano-pillar-patterned sapphire with different offcut angle by metalorganic vapor phase epitaxy". University of Bath Research Data Archive. $\quad$ https://doi.org/10.15125/BATH-XXXXX (Link to be finalised during proof stage).

[29] O. Reentilä, F. Brunner, A. Knauer, A. Mogilatenko, W. Neumann, H. Protzmann, M. Heuken, M. Kneissl, M. Weyers, and G. Tränkle, J. Crystal Growth 310, 1515 (2008).

[30] N. Susilo, J. Enslin, L. Sulmoni, M. Guttmann, U. Zeimer, T, Wernicke, M. Weyers, and M. Kneissl, Phys. Stat. Sol. A 215, 1700643 (2018).

[31] L. O. Nyakiti, J. Chaudhuri, E. A. Kenik, P. Lu, and J. H. Edgar, Mater. Res. Soc. Symp. Proc. 1040, Q11-03 (2008).

[32] G. Naresh-Kumar, B. Hourahine, P. R. Edwards, A. P. Day, A. Winkelmann, A. J. Wilkinson, P. J. Parbrook, C. England, and C. Trager-Cowan, PRL 108, 135503 (2012).

[33] G. Naresh-Kumar, D. Thomson, M. Nouf-Allehiani, J. Bruckbauer, P. R. Edwards, B. Hourahine, R. W. Martin, and C. Trager-Cowan, Mater. Sci. Semicond. Process. 47, 44 (2016).

[34] E. Pascal, B. Hourahine, G. Naresh-Kumar, K. Mingard, and C. Trager-Cowan, materialstoday: Proc. 5, 14652 (2018).

[35] G. Kusch, M. Nouf-Allehiani, F. Mehnke, C. Kuhn, P. R. Edwards, T. Wernicke, A. Knauer, V. Kueller, G. NareshKumar, M. Weyers, M. Kneissl, C. Trager-Cowan, and R. W. Martin, Appl. Phys. Lett. 107, 072103 (2015).

[36] I. Bryan, Z. Bryan, S. Mita, A. Rice, J. Tweedie, R. Collazo, and Z. Sitar, J. Crystal Growth. 438, 81 (2016).

[37] K. Bellmann, U.W. Pohl, C. Kuhn, T. Wernicke, and M. Kneissl, J. Crystal Growth 478, 187 (2017).

[38] P. Gay, P.B. Hirsch, and A. Kelly, Acta Metall. 1, 315 (1953).

[39] S.R. Lee, A.M. West, A.A. Allerman, K.E. Waldrip, D.M. Follstaedt, P.P. Provencio, D.D. Koleske, and C.R. Abernathy, Appl. Phys. Lett. 86, 241904 (2005).

[40] T. M. Katona, P. Cantu, S. Keller, Y. Wu, J. S. Speck, and S. P. DenBaars, Appl. Phys. Lett. 84, 5025 (2004).

[41] J. L. Weyher, Superlattices and Microstructures 40, 279 (2006).

[42] P. M. Anderson, J. P. Hirth, and J. Lothe, "Theory of Dislocations", Cambridge University Press (2017).
[43] A. Alasmari and G. Kusch, 2019. University of Strathclyde Datasets. https://doi.org/10.15129/XXXXX (Link to be finalised during proof stage).

[44] J. E. Northrup, L. T. Romano, and J. Neugebauer, Appl. Phys. Lett. 74, 2319 (1999).

[45] C. B. Soh, W. Liu, S. J. Chua, S. Tripathy, D. Z. Chi, J. Crystal Growth 268, 478 (2004).

[46] I.-H. Kim, H.-S. Park, Y.-J. Park, and T. Kim, Appl. Phys. Lett. 73, 1634 (1998).

[47] U. Zeimer, V. Kueller, A. Knauer, A. Mogilatenko, M. Weyers, and M. Kneissl, J. Cryst. Growth 377, 32 (2013).

[48] C. B. Soh, S. J. Chua, S. Tripathy, W. Liu, and D. Z. Chi, J. Phys.: Condens. Matter 17, 729 (2005).

[49] S. Walde, M. Brendel, U. Zeimer, F. Brunner, S. Hagedorn, and M. Weyers, J. Appl. Phys. 123, 161551 (2018). 